\title{
Dispersion relations and dynamic characteristics of bound states in the model of a Dirac field interacting with a material plane
}

\author{
Yury Pismak ${ }^{1, *}$ and Franz Wegner ${ }^{2}$ \\ ${ }^{1}$ Department of Elementary Particles and High Energy Physics, Saint-Petersburg State University, \\ 198504, St.Peterburg, Russia \\ ${ }^{2}$ Institute for Theoretical Physics, Ruprecht Karls University Heidelberg, D-69120, Heidelberg, \\ Germany
}

\begin{abstract}
Symanzik's approach for construction of quantum field model in inhomogeneous space-time is used as a basis for modeling the interaction of a macroscopic material body with quantum fields. In quantum electrodynamics it enables one to establish the most general form of the action functional describing the interaction of 2-dimensional material objects with photon and fermion fields. Results obtained within this approach for description of the interaction of the spinor field with a material plane are presented.
\end{abstract}

\section{Introduction}

Macro-manifestations of the quantum nature of vacuum have long attracted attention of many researchers. They become essential at distances from 10 to 1000 nanometers and form a special nanophysics, in which the quantum and classical features of the systems are combined. A well-known example of the nano-physical effect is the attraction of plates of an uncharged flat capacitor, theoretically predicted by Casimir in 1948 [1] and confirmed experimentally in the present time with a high degree of accuracy. Knowledge of the laws of nanophysics is becoming more and more relevant for the creation of new technical devices. Therefore, theoretical and experimental investigations of nanophysics are receiving increasing attention in recent years [2].

Within the framework of the approach proposed by Casimir, the interaction of capacitor plates with a vacuum is described by boundary conditions in the quantum field model [1]. An alternative approach to modeling the interaction of an extended material object with a quantum field is possible on the basis of the method proposed by K. Symanzik for construction of quantum field models in an inhomogeneous space-time [3]. It was developed for the description of interaction of the fields of quantum electrodynamics (QED) with extended material objects [4]. Such an approach makes it possible to describe quantitatively many physical phenomena within the framework of one universal model with a small number of parameters [4-11]. In the present paper we formulate general principles that have been used to construct models for the interaction of QED fields with two-dimensional material objects within the framework of the Symanzik approach and present the latest results obtained by us

*e-mail: ypismak@gmail.com 
in investigating the problem of bound states of Dirac particles on a homogeneous isotropic plane.

\section{Formulation of model}

The approach proposed by Symanzik for the description of quantum fields in vacuum with space-time inhomogeneities was used for modeling the interaction of the fields of quantum electrodynamics (QED) with extended material objects. It was based on the modification of the QED action functional

$$
S_{Q E D}(\bar{\psi}, \psi, A)=-\frac{1}{4} F_{\mu \nu} F^{\mu v}+\bar{\psi}(i \hat{\partial}-m+e \hat{A}) \psi, F_{\mu \nu}=\partial_{\mu} A_{v}-\partial_{\nu} A_{\mu}
$$

by an additional term $S_{d e f}(\bar{\psi}, \psi, A)$ (defect action) describing the interaction of the material body with the photon field $A_{\mu}$ and Dirac fields $\bar{\psi}, \psi$.

From the basic principles of QED ( gauge invariance, locality, renormalizability ) it follows that for thin film without charges and currents, whose shape is defined by equation $\Phi(x)=0, x=\left(x_{0}, x_{1}, x_{2}, x_{3}\right)$, the defect action reads [4-11]

$$
S_{d e f}(\bar{\psi}, \psi, A)=S_{d e f}(A, \Phi)+S_{d e f}(\bar{\psi}, \psi, \Phi)+S_{d e f}(\Phi) .
$$

The action $S_{d e f}(A, \Phi)$ is a surface Chern-Simon action

$$
S_{d e f}(A, \Phi)=\frac{a}{2} \int \varepsilon^{\lambda \mu \nu \rho} \partial_{\lambda} \Phi(x) A_{\mu}(x) F_{v \rho}(x) \delta(\Phi(x)) d x,
$$

where $\varepsilon^{\lambda \mu v \rho}$ denotes the totally antisymmetric tensor $\left(\varepsilon^{0123}=1\right), a$ is a constant dimensionless parameter. The fermion defect action can be written as

$$
S_{d e f}(\bar{\psi}, \psi, \Phi)=\int \bar{\psi}(x)\left[\lambda+u_{\mu} \gamma^{\mu}+\gamma^{5}\left(\tau+v_{\mu} \gamma^{\mu}\right)+\omega_{\mu \nu} \sigma^{\mu v}\right] \psi(x) \delta(\Phi(x)) d x .
$$

Here, $\gamma^{\mu}, \mu=0,1,2,3$, are the Dirac matrices, $\gamma^{5}=i \gamma^{0} \gamma^{1} \gamma^{2} \gamma^{3}, \sigma^{\mu v}=i\left(\gamma^{\mu} \gamma^{\nu}-\gamma^{\gamma} \gamma^{\mu}\right) / 2$, and $\lambda, \tau, u_{\mu}, v_{\mu}, \omega^{\mu \nu}=-\omega^{\nu \mu}, \mu, v=0,1,2,3$ are 16 dimensionless parameters. The defect action $S_{d e f}(\Phi)$ independent from the QED fields is necessary for the cancelation of ultraviolet divergences in the renormalized Casimir energy density.

It is the most general form of a gauge invariant action concentrated on the defect surface which is invariant in respect to reparametrization and independent from parameters with negative dimensions.

The full action of the model, which satisfies the requirement of locality, gauge invariance and renormalizability, has the form

$$
S(\bar{\psi}, \psi, A, \Phi)=S_{Q E D}(\bar{\psi}, \psi, A)+S_{d e f}(A, \Phi)+S_{d e f}(\bar{\psi}, \psi, \Phi)+S_{d e f}(\Phi) .
$$

Due to the requirements of renormalizability the field interaction is described by the standard contribution $e \bar{\psi} \hat{A} \psi$ to the QED action.

\section{Casimir energy for two parallel planes}

The energy density $E_{C a s}$ of the Casimir effect for two parallel planes with Chern-Simons defect action describing the interaction of them with the photon field, is calculated in [4]. It is expressed in an explicit form in terms of polylogarithm function $\mathrm{Li}_{4}(x)$ in the following way:

$$
E_{C a s}=-\frac{1}{16 \pi^{2} \mathrm{r}^{3}} \sum_{k=1}^{2} \operatorname{Li}_{4}\left(\frac{a_{1} a_{2}}{a_{1} a_{2}+i(-1)^{k}\left(a_{1}+a_{2}\right)-1}\right) .
$$


Here $E_{C a s}$ is an energy density of interaction of quantum vacuum fluctuations with two parallel planes, depending on the distance $r$ between them and Chern-Simons coupling constants $a_{1}, a_{2}$. The function $\mathrm{Li}_{4}(x)$ is defined as

$$
\mathrm{Li}_{4}(x)=\sum_{k=1}^{\infty} \frac{x^{k}}{k^{4}}=-\frac{1}{2} \int_{0}^{\infty} k^{2} \ln \left(1-x e^{-k}\right) d k .
$$

For identical defect planes $\left(a_{1}=a_{2}=a\right)$ the Casimir pressure $P_{C a s}(\mathrm{r}, a)$ on them is given by

$$
P_{C a s}(\mathrm{r}, a)=-\frac{\partial E_{C a s}(\mathrm{r}, a)}{\partial \mathrm{r}}=-\frac{\pi^{2}}{240 \mathrm{r}^{4}} f(a)
$$

The function $f(a)$ is even ( $\mathrm{f}(\mathrm{a})=\mathrm{f}(-\mathrm{a})$ ), has the minimum $f\left(a_{m}\right)=-0,11723$ at $a_{m}=0,5892$, and $f\left(a_{0}\right)=f(0)=0$ at $a_{0}=1,03246, \lim _{a \rightarrow \infty} f(a)=1$. For $f(a)=1$ the right hand side of (2) agrees with the result obtained by Casimir for two parallel plates from high conducting material. For $|a|>a_{0}$, the Casimir force is attractive and it is repulsive for $a_{0}<0$.

\section{Statement of problem for Dirac field}

If one considers the material plane $x_{3}=0$ as a defect changing the QED vacuum, then in the Dirac part of the action (1)

$$
S_{D}(\bar{\psi}, \psi)=\int \bar{\psi}(x)\left(i \hat{\partial}-m+\Omega\left(x_{3}\right)\right) \psi(x) d x
$$

the interaction of the spinor field with the plane is described by $\Omega\left(x_{3}\right)=Q \delta\left(x_{3}\right)$. Since $\Omega\left(x_{3}\right)$ and $\delta\left(x_{3}\right)$ have the dimension of mass, the matrix $Q$ is dimensionless. For a homogeneous isotropic material the matrix $Q$ can generally be presented in the form

$$
Q=r_{1} I+i r_{2} \gamma^{5}+r_{3} \gamma^{3}+r_{4} \gamma^{5} \gamma^{3}+r_{5} \gamma^{0}+r_{6} \gamma^{5} \gamma^{0}+i r_{7} \gamma^{0} \gamma^{3}+i r_{8} \gamma^{1} \gamma^{2},
$$

where $I$ is the identity $4 \times 4$ matrix and $\gamma^{j}, j=0,1,2,3$, are the Dirac matrices, $\gamma^{5}=i \gamma^{0} \gamma^{1} \gamma^{2} \gamma^{3}$.

Movement of spinor particle in the field of defect $\Omega\left(x_{3}\right)$ is described by a modified Dirac equation

$$
\left(i \hat{\partial}-m+\Omega\left(x_{3}\right)\right) \psi(x)=0 .
$$

It is one of the Euler-Lagrange equations, which is obtained by variational differentiation of the action $S_{D}(\bar{\psi}, \psi)(3)$ over $\bar{\psi}(x)$. Differentiating over $\psi(x)$ we obtain the second equation

$$
\left(\partial_{\mu} \bar{\psi}(x)\right) \gamma^{\mu}+\bar{\psi}(x)\left(m-\Omega\left(x_{3}\right)\right)=0 .
$$

The condition $\bar{\psi}(x)=\psi^{*}(x) \gamma_{0}$ is fulfilled if $\gamma_{0} \Omega^{+}(x)=\Omega(x) \gamma_{0}$. This holds for real values of parameters $r_{j}, j=1, \ldots, 8$.

Let us introduce convenient notations. For $2 \times 2$-matrices $M$ with elements $M_{i j}, i, j=1,2$, we define the $4 \times 4$-matrices $M^{(+)}, M^{(-)}$in the following way

$$
M^{(+)}=\left(\begin{array}{cccc}
M_{11} & 0 & M_{12} & 0 \\
0 & 0 & 0 & 0 \\
M_{21} & 0 & M_{22} & 0 \\
0 & 0 & 0 & 0
\end{array}\right), M^{(-)}=\left(\begin{array}{cccc}
0 & 0 & 0 & 0 \\
0 & M_{11} & 0 & M_{12} \\
0 & 0 & 0 & 0 \\
0 & M_{21} & 0 & M_{22}
\end{array}\right) .
$$

Let $\mathcal{M}^{( \pm)}$be the set of all the matrices $M^{( \pm)}$, then one has for arbitrary matrices $M_{1}^{( \pm)}, M_{2}^{(\mp)}$,

$$
M_{1}^{( \pm)} M_{2}^{(\mp)}=0, M_{1}^{( \pm)} M_{2}^{( \pm)} \in \mathcal{M}^{( \pm)} .
$$


If we denote by $\tau_{0}$ the unit $2 \times 2$ - matrix, and by $\tau_{j}, j=1,2,3$, the Pauli matrices, and $\tau_{\mu}^{(+)}, \tau_{\mu}^{(-)}, \mu=0,1,2,3$, the corresponding $4 \times 4$-matrices, then the matrix $Q$ can be presented as

$$
Q=Q^{(+)}+Q^{(-)}, Q^{( \pm)}=\sum_{j=0}^{3} q_{j}^{( \pm)} \tau_{j}^{( \pm)},
$$

where $q_{0}^{( \pm)}=r_{18}^{ \pm}, q_{1}^{( \pm)}=i r_{27}^{ \pm}, q_{2}^{( \pm)}= \pm i r_{36}^{\mp}, q_{3}^{( \pm)}=\mp r_{45}^{\mp}$, and $r_{i j}^{ \pm}=r_{i} \pm r_{j}$.

We denote the solution of the modified Dirac equation as $\psi(x)$, and also $\psi_{-}(x) \equiv \psi(x)$ for $x_{3}<0, \psi_{+}(x) \equiv \psi(x)$ for $x_{3}>0$. The spinors $\psi(x)_{ \pm}$for $x_{3} \neq 0$ satisfy the free Dirac equation and the boundary condition

$$
\lim _{x^{3} \rightarrow+0} \psi_{+}(x)=S \lim _{x^{3} \rightarrow-0} \psi_{-}(x) .
$$

One can choose the regularization procedure for $\delta\left(x_{3}\right)$ in such a way that the matrix $S$ is expressed in terms of $Q$ as

$$
S=\exp \left\{-i \gamma^{3} Q\right\}
$$

It follows from $Q^{\dagger}=\gamma_{0} Q \gamma_{0}$ that $S^{\dagger} \gamma^{0} \gamma^{3} S=\gamma^{0} \gamma^{3}$, where $S^{\dagger}$ is the hermitian conjugated matrix $S$, and the matrix $S$ can be presented as $S=S^{(+)}+S^{(-)}$, where

$$
S^{( \pm)}=e^{i \chi_{ \pm}}\left(\varsigma_{0 \pm} \tau_{0}^{( \pm)}+i \varsigma_{1 \pm} \tau_{1}^{( \pm)}+\varsigma_{2 \pm} \tau_{2}^{( \pm)}+\varsigma_{3 \pm} \tau_{3}^{( \pm)}\right), \varsigma_{0 \pm}^{2}+\varsigma_{1 \pm}^{2}-\varsigma_{2 \pm}^{2}-\varsigma_{3 \pm}^{2}=1 .
$$

Here, $\chi_{ \pm}$and $\varsigma_{\mu \pm}, \mu=0,1,2,3$ are real parameters which can be expressed in terms of the parameters $r_{j}, 1 \leq j \leq 8$, of the matrix $Q$.

\section{Free Dirac equations}

The free Dirac equation in coordinate space reads

$$
(i \hat{\partial}-m) \psi(x)=0 .
$$

By substitution $\psi(x)$ in the form

$$
\psi(x)=\frac{1}{(2 \pi)^{4}} \int e^{-i p x} \psi(\bar{p}) d \bar{p}, \bar{p}=\left(p^{0}, p^{1}, p^{2}\right), p_{3}=\sqrt{p_{0}^{2}-m^{2}-p_{1}^{2}-p_{2}^{2}},
$$

one obtains

$$
(\hat{p}-m) \psi(\bar{p})=0 .
$$

The spinor $\psi(x)$ (4) describes a scattering state for real $p_{3}$ and a bound state if $p_{3}$ is imaginary.

The general solution $\psi(\bar{p})$ of the Dirac equation (5) can be presented as an arbitrary linear combination of linear independent spinors $\psi_{1}(\bar{p}), \psi_{2}(\bar{p})$ for $p_{0}>0$ (Dirac particle), and $\psi_{1}^{\prime}(\bar{p}), \psi_{2}^{\prime}(\bar{p})$ for $p_{0}<0$ (Dirac anti-particle);

$$
\psi_{1}(\bar{p})=\left\{\begin{array}{c}
1 \\
0 \\
\frac{-p_{3}}{m+p_{0}} \\
\frac{-p_{1}-i p_{2}}{m+p_{0}}
\end{array}\right\}, \psi_{2}(\bar{p})=\left\{\begin{array}{c}
0 \\
1 \\
\frac{-p_{1}+i p_{2}}{m+p_{0}} \\
\frac{p_{3}}{m+p_{0}}
\end{array}\right\}, \psi_{1}^{\prime}(\bar{p})=\left\{\begin{array}{c}
\frac{p_{1}-i p_{2}}{m-p_{0}} \\
\frac{p_{3}}{p_{0}-m} \\
0 \\
1
\end{array}\right\}, \psi_{2}^{\prime}(\bar{p})=\left\{\begin{array}{c}
\frac{p_{3}}{m-p_{0}} \\
\frac{p_{1}+i p_{2}}{m-p_{0}} \\
1 \\
0
\end{array}\right\} .
$$


Substituting $p_{3} \rightarrow \pm i \kappa$ with $\kappa=|\kappa|=\sqrt{m^{2}+p_{1}^{2}+p_{2}^{2}-p_{0}^{2}}$ we obtain the spinors describing the bound states for $x_{3} \geq 0$ and $x_{3} \leq 0: \psi_{ \pm}(\bar{p})=\left.\psi(\bar{p})\right|_{p_{3} \rightarrow \mp i \kappa}, \psi_{ \pm}^{\prime}(\bar{p})=\left.\psi^{\prime}(\bar{p})\right|_{p_{3} \rightarrow \mp i \kappa}$. They can be presented as follows

$$
\begin{array}{r}
\psi_{+}(\bar{p})=a_{1} \psi_{1+}(\bar{p})+a_{2} e^{i \phi} \psi_{2+}(\bar{p}), \psi_{-}(\bar{p})=d_{1} \psi_{1-}(\bar{p})+d_{2} e^{i \phi} \psi_{2-}(\bar{p}), \\
\psi_{+}^{\prime}(\bar{p})=a_{1}^{\prime} \psi_{1+}^{\prime}(\bar{p})+a_{2}^{\prime} e^{i \phi} \psi_{2+}^{\prime}(\bar{p}), \psi_{-}^{\prime}(\bar{p})=d_{1}^{\prime} \psi_{1-}^{\prime}(\bar{p})+d_{2}^{\prime} e^{i \phi} \psi_{2-}^{\prime}(\bar{p}), \\
\psi_{j \pm}(\bar{p})=\left.\psi_{j}(\bar{p})\right|_{p_{3} \rightarrow \mp i \kappa}, \psi_{j \pm}^{\prime}(\bar{p})=\left.\psi_{j}^{\prime}(\bar{p})\right|_{p_{3} \rightarrow \mp i \kappa}, j=1,2 .
\end{array}
$$

Here

$$
\begin{gathered}
\psi_{1 \pm}(\bar{p})=\left\{\begin{array}{c}
1 \\
0 \\
\pm i k \\
f e^{i \phi}
\end{array}\right\}, \psi_{2 \pm}(\bar{p})=\left\{\begin{array}{c}
0 \\
1 \\
f e^{-i \phi} \\
\mp i k
\end{array}\right\}, \psi_{1 \pm}^{\prime}(\bar{p})=\left\{\begin{array}{c}
-f e^{-i \phi} \\
\pm i k \\
0 \\
1
\end{array}\right\}, \psi_{2 \pm}^{\prime}(\bar{p})=\left\{\begin{array}{c}
\mp i k \\
-f e^{i \phi} \\
1 \\
0
\end{array}\right\}, \\
k=\frac{\kappa}{m+\left|p_{0}\right|}, \frac{p^{1}+i p^{2}}{m+\left|p_{0}\right|}=-\frac{p_{1}+i p_{2}}{m+\left|p_{0}\right|}=f e^{i \phi}, f=|f| .
\end{gathered}
$$

The spinors $\psi_{ \pm}, \psi_{ \pm}^{\prime}$ fulfill the relations

$$
\psi_{+}(\bar{p})=S \psi_{-}(\bar{p}), \psi_{+}^{\prime}(\bar{p})=S \psi_{-}^{\prime}(\bar{p}), S=e^{-i \gamma^{3} Q} .
$$

which can be presented as systems of linear equations for the coefficients $a_{1}, a_{2}, d_{1}, d_{2}$, $a_{1}^{\prime}, a_{2}^{\prime}, d_{1}^{\prime}, d_{2}^{\prime}$ contained in $\psi_{ \pm}, \psi_{ \pm}^{\prime}$.

\section{Properties of bound states}

For $p_{0}>0$, we consider the quantities of the form

$$
\bar{\psi}_{ \pm}\left(\bar{p}, x_{3}\right) \Gamma \psi_{ \pm}\left(\bar{p}, x_{3}\right)=\bar{\psi}_{ \pm}(\bar{p}) \Gamma \psi_{ \pm}(\bar{p}) e^{-2 \kappa\left|x_{3}\right|}
$$

with $4 \times 4$ basic Dirac matrices $\Gamma$ using convenient notations

$$
\begin{array}{r}
N_{0+}=a_{1}^{*} a_{1}+a_{2}^{*} a_{2}, N_{1+}=a_{1}^{*} a_{2}+a_{2}^{*} a_{1}, N_{2+}=a_{1}^{*} a_{1}-a_{2}^{*} a_{2}, N_{3+}=i\left(a_{1}^{*} a_{2}-a_{2}^{*} a_{1}\right), \\
N_{0-}=d_{1}^{*} d_{1}+d_{2}^{*} d_{2}, N_{1-}=d_{1}^{*} d_{2}+d_{2}^{*} d_{1}, N_{2-}=d_{1}^{*} d_{1}-d_{2}^{*} d_{2}, N_{3-}=i\left(d_{1}^{*} d_{2}-d_{2}^{*} d_{1}\right), \\
\vec{\gamma}=\left\{\gamma^{1}, \gamma^{2}, \gamma^{3}\right\}, \vec{\sigma}=\left\{\sigma^{1}, \sigma^{2}, \sigma^{3}\right\}, \sigma^{j}=\sum_{k, l=1}^{3} \frac{i}{2} \varepsilon^{j k l} \gamma^{k} \gamma^{l}, j=1,2,3,
\end{array}
$$

where $\varepsilon^{j k l}$ is totally antisymmetric and $\varepsilon^{123}=1$.

For scalar and pseudoscalar invariant densities

$$
\bar{\psi}_{ \pm}(x) \psi_{ \pm}(x)=e^{-2 \kappa\left|x^{3}\right|} \rho_{ \pm}(\bar{p}), \bar{\psi}_{ \pm}(x) \gamma^{5} \psi_{ \pm}(x)=i e^{-2 \kappa\left|x^{3}\right|} \rho_{5 \pm}(\bar{p}),
$$

and for components of electric and axial 4-currents

$$
\bar{\psi}(x) \gamma^{\mu} \psi(x)=e^{-2 \kappa\left|x^{3}\right|} j_{ \pm}^{\mu}(\bar{p}), \bar{\psi}(x) \gamma^{\mu} \gamma^{5} \psi(x)=e^{-2 \kappa\left|x^{3}\right|} j_{5 \pm}^{\mu}(\bar{p}), \mu=0,1,2,3,
$$

we obtain the following results

$$
\begin{array}{r}
\rho_{ \pm}=N_{0 \pm}\left(1-f^{2}-k^{2}\right) \pm 2 N_{3 \pm} f k, j_{ \pm}^{0}=N_{0 \pm}\left(1+f^{2}+k^{2}\right) \mp 2 N_{3 \pm} f k, j_{ \pm}^{3}=0, \\
j_{ \pm}^{1}=j_{ \pm}^{\|} \cos (\phi)+j_{ \pm}^{\perp} \sin (\phi), j_{ \pm}^{2}=j_{ \pm}^{\|} \sin (\phi)-j_{ \pm}^{\perp} \cos (\phi), \\
j_{ \pm}^{\|}=2\left(N_{0 \pm} f \mp N_{3 \pm} k\right), j_{ \pm}^{\perp}= \pm 2 N_{1 \pm} k, \\
\rho_{5 \pm}= \pm 2 N_{2 \pm} k, j_{5 \pm}^{0}=2 N_{1 \pm} f, j_{5 \pm}^{3}=N_{2 \pm}\left(1-f^{2}+k^{2}\right), \\
j_{5 \pm}^{1}=j_{5 \pm}^{\|} \cos (\phi)+j_{5 \pm}^{\perp} \sin (\phi), j_{5 \pm}^{2}=j_{5 \pm}^{\|} \sin (\phi)-j_{5 \pm}^{\perp} \cos (\phi), \\
j_{5 \pm}^{\|}=N_{1 \pm}\left(1+f^{2}-k^{2}\right), j_{5 \pm}^{\perp}=N_{3 \pm}\left(1-f^{2}-k^{2}\right) \pm 2 N_{0 \pm} f k
\end{array}
$$


The vectors $\vec{\epsilon}_{ \pm}(\bar{p}), \vec{\mu}_{ \pm}(\bar{p})$ we define in the following way:

$$
\bar{\psi}_{ \pm}(x) \gamma^{0} \vec{\gamma} \psi_{ \pm}(x)=i e^{-2 \kappa\left|x^{3}\right|} \vec{\epsilon}_{ \pm}(\bar{p}), \bar{\psi}_{ \pm}(x) \vec{\sigma} \psi_{ \pm}(x)=e^{-2 \kappa\left|x^{3}\right|} \vec{\mu}_{ \pm}(\bar{p}) .
$$

They characterize the electric and magnetic dipole moments of the bound state and can be presented as

$$
\begin{array}{r}
\epsilon_{ \pm}^{1}=\epsilon_{ \pm}^{\perp} \sin (\phi), \epsilon_{ \pm}^{2}=-\epsilon_{ \pm}^{\perp} \cos (\phi), \epsilon^{3}=-2\left(N_{3 \pm} f \mp N_{0 \pm} k\right), \epsilon^{\perp}=2 f N_{2 \pm}, \\
\mu_{ \pm}^{1}=\mu_{ \pm}^{\|} \cos (\phi)+\mu_{ \pm}^{\perp} \sin (\phi), \mu_{ \pm}^{2}=\mu_{ \pm}^{\|} \sin (\phi)-\mu_{ \pm}^{\perp} \cos (\phi), \mu_{ \pm}^{3}=N_{2 \pm}\left(1-k^{2}+f^{2}\right), \\
\mu_{ \pm}^{\|}=N_{1 \pm}\left(1+k^{2}-f^{2}\right), \mu_{ \pm}^{\perp}=N_{3 \pm}\left(1+f^{2}+k^{2}\right) \mp 2 N_{0 \pm} f k .
\end{array}
$$

We see that there are relations between these quantities which are independent from the parameters of the model:

$$
\begin{aligned}
& j_{ \pm}^{0} p^{0}-j_{ \pm}^{1} p^{1}-j_{ \pm}^{2} p^{2}=m \rho_{ \pm}, j_{5 \pm}^{0} p^{0}-j_{5 \pm}^{1} p^{1}-j_{5 \pm}^{2} p^{2}=0, m \rho_{5 \pm}= \pm \kappa j_{5 \pm}^{3} \\
& \vec{\epsilon}_{ \pm} \vec{\mu}_{ \pm}=\rho_{ \pm} \rho_{5 \pm}, \vec{\epsilon}_{ \pm}^{2}-\vec{\mu}_{ \pm}^{2}=\rho_{5 \pm}^{2}-\rho_{ \pm}^{2}, m j_{ \pm}^{0}-p^{0} \rho_{ \pm}= \pm \kappa \epsilon_{ \pm}^{3}, p^{0} j_{5 \pm}^{3}=m \mu_{3 \pm}
\end{aligned}
$$

and all the factors $N_{j \pm}$ are expressed in terms of densities $\rho_{ \pm}, \rho_{5 \pm}$ and charges $j_{ \pm}^{0}, j_{5 \pm}^{0}$ :

$$
N_{0 \pm}=\frac{\rho_{ \pm}+j_{ \pm}^{0}}{2}, N_{1 \pm}=\frac{j_{5 \pm}^{0}}{2 f}, N_{2 \pm}= \pm \frac{\rho_{5 \pm}}{2 k}, N_{3 \pm}= \pm \frac{\left(\rho_{ \pm}+j_{ \pm}^{0}\right)\left(f^{2}+k^{2}\right)+\rho_{ \pm}-j_{ \pm}^{0}}{4 f k}
$$

\section{Equations for amplitudes}

Let us denote

$$
\begin{array}{r}
a=\left\{\begin{array}{l}
a_{1} \\
a_{2}
\end{array}\right\}, d=\left\{\begin{array}{l}
d_{1} \\
d_{2}
\end{array}\right\}, S_{ \pm}=\left(\begin{array}{cc}
\varsigma_{0 \pm}+\varsigma_{3 \pm} & i\left(\varsigma_{1 \pm}-\varsigma_{2 \pm}\right) \\
i\left(\varsigma_{1 \pm}+\varsigma_{2 \pm}\right) & \varsigma_{0 \pm}-\varsigma_{3 \pm}
\end{array}\right), \\
K_{+}=\frac{1}{f}\left(\begin{array}{cc}
f & 0 \\
-i k & 1
\end{array}\right), K_{-}=\frac{1}{f}\left(\begin{array}{cc}
i k & 1 \\
f & 0
\end{array}\right), L_{+}=\left(\begin{array}{cc}
1 & 0 \\
-i k & f
\end{array}\right), L_{-}=\left(\begin{array}{cc}
0 & 1 \\
f & i k
\end{array}\right) .
\end{array}
$$

It follows from $\psi_{+}=S \psi_{-}, S^{( \pm)}=e^{i \chi_{ \pm}} s_{ \pm}^{( \pm)}$that the vectors $a, d$ fulfill the equations

$$
a=e^{i \chi+} T_{+} d, a=e^{i \chi-} T_{-} d,
$$

where $T_{ \pm}=K_{ \pm} s_{ \pm} L_{ \pm}$. Hence, $d$ satisfies the homogeneous equation $T d=0$ with

$$
T=e^{i \chi^{\prime}}\left(e^{i \chi} T_{+}-e^{-i \chi} T_{-}\right), \chi=\frac{\chi_{+}-\chi_{-}}{2}, \chi^{\prime}=\frac{\chi_{+}+\chi_{-}}{2} .
$$

It has nontrivial solution, if $\operatorname{det} T=0$. The matrix $T$ can be presented in the form

$$
\begin{gathered}
T=\frac{e^{i \chi^{\prime}}}{f}\left(\begin{array}{cc}
t_{11} & t_{12} \\
t_{21} & t_{22}
\end{array}\right), \\
t_{11}=\left(e^{i \chi}\left(k\left(\varsigma_{1+}-\varsigma_{2+}\right)+\varsigma_{0+}+\varsigma_{3+}\right)+e^{-i \chi}\left(k\left(\varsigma_{1-}-\varsigma_{2-}\right)-\varsigma_{0-}+\varsigma_{3-}\right)\right) f, \\
t_{12}=i\left(e^{i \chi} f^{2}\left(\varsigma_{1+}-\varsigma_{2+}\right)+e^{-i \chi}\left(k^{2}\left(\varsigma_{1-}-\varsigma_{2-}\right)-2 k \varsigma_{0-}-\varsigma_{1-}-\varsigma_{2-}\right)\right), \\
t_{21}=-i\left(e^{i \chi}\left(k^{2}\left(\varsigma_{1+}-\varsigma_{2+}\right)+2 k \varsigma_{0+}-\varsigma_{1+}-\varsigma_{2+}\right)+e^{-i \chi} f^{2}\left(\varsigma_{1-}-\varsigma_{2-}\right)\right), \\
t_{22}=\left(e^{i \chi}\left(k\left(\varsigma_{1+}-\varsigma_{2+}\right)+\varsigma_{0+}-\varsigma_{3+}\right)+e^{-i \chi}\left(k\left(\varsigma_{1-}-\varsigma_{2-}\right)-\varsigma_{0-}-\varsigma_{3-}\right)\right) f .
\end{gathered}
$$


Thus, the solvability condition of equation $T d=0$ can be written in the form

$$
\begin{array}{r}
t_{11} t_{22}-t_{12} t_{21}=2 f^{2}( \\
\left.\cos (2 \chi)-\varsigma_{0-} \varsigma_{0+}+\varsigma_{1-} \varsigma_{1+}-\varsigma_{2-} \varsigma_{2+}-\varsigma_{3-} \varsigma_{3+}\right)- \\
-\left(\left(k^{2}-f^{2}\right)\left(\varsigma_{1-}-\varsigma_{2-}\right)-2 k \varsigma_{0-}-\varsigma_{1-}-\varsigma_{2-}\right) \times \\
\times\left(\left(k^{2}-f^{2}\right)\left(\varsigma_{1+}-\varsigma_{2+}\right)+2 k \varsigma_{0+}-\varsigma_{1+}-\varsigma_{2+}\right)=0 .
\end{array}
$$

The general solution of equations (6) for $a, d$ can be presented as follows

$$
d=c\left\{\begin{array}{c}
t_{12} \\
-t_{11}
\end{array}\right\}, a=c^{\prime}\left\{\begin{array}{c}
t_{12}^{\prime} \\
-t_{11}^{\prime}
\end{array}\right\}
$$

Here, $c$ is an arbitrary constant and

$$
\begin{gathered}
t_{12}^{\prime}=\left.t_{12}\right|_{\chi \rightarrow-\chi}, k \rightarrow-k, \varsigma_{0 \pm} \rightarrow-\varsigma_{0 \pm}, t_{11}^{\prime}=\left.t_{11}\right|_{\chi \rightarrow-\chi}, k \rightarrow-k, \varsigma_{0 \pm} \rightarrow-\varsigma_{0 \pm}, \\
c^{\prime}=c \frac{e^{i \chi^{\prime}}\left(h_{1} g_{2}-g_{1} h_{2}\right)}{g_{2} e^{i \chi}-h_{2} e^{-i \chi}}, h_{1}=\varsigma_{0+}+\varsigma_{3+}+k\left(\varsigma_{1+}-\varsigma_{2+}\right), h_{2}=f^{2}\left(\varsigma_{1+}-\varsigma_{2+}\right), \\
g_{1}=\varsigma_{0-}-\varsigma_{3-}-k\left(\varsigma_{1-}-\varsigma_{2-}\right), g_{2}=\varsigma_{1-}+\varsigma_{2-}+2 k \varsigma_{0-}-k^{2}\left(\varsigma_{1-}-\varsigma_{2-}\right) .
\end{gathered}
$$

\section{Dispersion relation}

The solvability condition of equations (6) $\operatorname{det} T=0$ (dispersion relation) can be presented as

$$
\begin{aligned}
& \left(p_{1}^{2}+p_{2}^{2}\right)\left(\cos (2 \chi)-\varsigma_{0-} \varsigma_{0+}+\varsigma_{1-} \varsigma_{1+}-\varsigma_{2-} \varsigma_{2+}-\varsigma_{3-} \varsigma_{3+}\right)- \\
& -2\left(p_{0} \varsigma_{1-}+m \varsigma_{2-}+\kappa \varsigma_{0-}\right)\left(p_{0} \varsigma_{1+}+m \varsigma_{2+}-\kappa \varsigma_{0+}\right)=0 .
\end{aligned}
$$

In virtue of $p_{0}^{2}+\kappa^{2}-p_{1}^{2}-p_{2}^{2}-m^{2}=0$, it follows from the dispersion relation that $p_{0}, \kappa, m$ satisfy the equation

$$
\begin{array}{r}
\left(p_{0}^{2}+\kappa^{2}-\mu^{2}\right)\left(\cos \left(\chi_{+}-\chi_{-}\right)-\varsigma_{0-} \varsigma_{0+}+\varsigma_{1-} \varsigma_{1+}-\varsigma_{2-} \varsigma_{2+}-\varsigma_{3-} \varsigma_{3+}\right)- \\
-2\left(p_{0} \varsigma_{1-}+m \varsigma_{2-}+\kappa \varsigma_{0-}\right)\left(p_{0} \varsigma_{1+}+m \varsigma_{2+}-\kappa \varsigma_{0+}\right)=0
\end{array}
$$

which describes the relation between the dimensionless magnitudes $p_{0} / m, \kappa / m$ characterizing the bound state. Its solution is presented in the $\left(p_{0}, \kappa\right)$-plane by hyperbola or by two straight lines.

Since for Dirac particle the physical values of $p_{0}, \kappa$ are positive, the bound state can be realized if there are points of the $\left(p_{0}, \kappa\right)$-plot presenting the dispersion relation in the region $p_{0}>0, \kappa>0$. Moreover, since $p_{1}^{2}+p_{2}^{2} \geq 0$, the restriction $p_{0}^{2}+\kappa^{2} \geq m^{2}$ has to be obeyed. This part of plot can be connected or disconnected, and the possible values of $p_{0}, \kappa$ for bound state can be both restricted and non-restricted from above.

By $\varsigma_{0 \pm}=\varsigma_{2 \pm}=0$ the dispersion law has the form

$$
p_{0}^{2}-v_{F}^{2}\left(p_{1}^{2}+p_{2}^{2}\right)=0, v_{F}=\sqrt{\frac{\cos (2 \chi)+\varsigma_{1-} \varsigma_{1+}-\varsigma_{3-} \varsigma_{3+}}{2\left(\varsigma_{1-\varsigma_{1+}}\right)}}, \varsigma_{1 \pm}^{2}-\varsigma_{3 \pm}^{2}=1,
$$

and

$$
0 \leq v_{F} \leq 1, \kappa^{2}=\mu^{2}+\left(p_{1}^{2}+p_{2}^{2}\right)\left(1-v_{F}^{2}\right)
$$

It describes the propagation of massless particle in the defect plane with the Fermi-velocity $v_{F}$. The motion of such particles explains numerous effects in graphene. 


\section{Conclusions}

In the framework of the Symanzik approach, it is possible to build the model of QED field interaction with 2D material. The action of the model consist of the usual QED action and extra defect contribution. The action contains parameters, that characterize the material property. The Casimir pressure and Casimir-Polder potential appears to be non-universal and dependent on the properties of material [4], [5]. The Casimir force can be both attractive and repulsive [4].

The characteristics of photons and Dirac particles scattering on the defect plane can be calculated in the model, also the properties of states localized near the defect plane can be investigated [6-11].

The models obtained on these basis results could be used for the theoretical description of the interaction of electrons, positrons and neutrons with two-dimensional materials (graphene, thin films, sputters, sharp boundaries of a solid body). Simple modifications of the model allows to take into account the effects of external electromagnetic fields.

\section{Acknowledgments}

Yu.P. is grateful to Russian Foundation for Basic Research for financial support in frameworks of grant No. 16-02-00943-a.

\section{References}

[1] H. B. G Casimir, Proc. K. Ned. Akad. Wet B. 51, 793-795 (1948).

[2] M. Bordag, G. L. Klimchitskaya, U. Mohideen, and V. M. Mostepanenko, Advances in the Casimir Effect, Intl. Ser. Monogr. Phys., Vol. 145 (Oxford Univ. Press, Oxford 2009); G. L. Klimchitskaya, U. Mohideen and V. M. Mostepanenko, Rev. Mod. Phys. 81, 18271885 (2009).

[3] K. Symanzik, Nucl.Phys. B 190, 1-44 (1981).

[4] V. N. Markov and Yu. M. Pis'mak, J. Phys. A: Math. Gen. 39, 6525-6532 (2006); arXiv: hep-th/0505218v3 (2005).

[5] V. N. Marachevsky and Yu. M. Pis'mak, Phys. Rev. D 81, 065005 (2010); arXiv:0907.1985v2 [hep-th] (2009).

[6] D. Yu. Pis'mak and Yu. M. Pis'mak, Theor. Math. Phys. 175,816-826 (2013); Phys. Part. Nuclei 44, 450-461 (2013); Theor. Math. Phys. 166, 1423-1431 (2011).

[7] D. Yu. Pis'mak, Yu. M. Pis'mak and F. J. Wegner, Phys. Rev. E 92, 013204 (2015); arXiv:1406.1598v1 [hep-th] (2014).

[8] I. V. Fialkovsky, V. N. Markov and Yu. M. Pis'mak, J. Phys. A: Math. Gen. 39, 6357-6363 (2006); Int. J. Mod. Phys. A 21, 2601-2616 (2006); arXiv:hep-th/0311236v2 (2003).

[9] D. Yu. Pismak and Yu. M. Pismak, Theor. Math. Phys. 184, 3, 1329-1341 (2015).

[10] Yu. M. Pismak and D. Yu. Shukhobodskaia, EPJ Web of Conferences 125, 05022 (2016), EPJ Web of Conferences 126, 05012 (2016), EPJ Web of Conferences 158, 07005 (2017)

[11] Yu. M. Pismak, Phys. Part. Nucl. Lett, 15, 380-383 (2018) 